\title{
CMS Tracker alignment and material budget measurements
}

\section{Ernesto Migliore*}

Dipartimento di Fisica Sperimentale, Università di Torino e INFN Sezione di Torino

E-mail: miglioreato.infn.it

The CMS Silicon Tracker consists of 1440 pixel modules and 15148 strip modules covering an area of about 200 square meters. To achieve an optimal track-parameter resolution, the position and orientation of the modules must be determined with a precision of a few microns and an accurate representation of the distribution of material in the Tracker is needed. Results of the alignment of the Tracker are presented, based on the analysis of data from cosmic ray muons and proton-proton collisions. The alignment is validated by data-driven studies and compared with predictions from a detailed detector simulation. Reconstructed photon conversions and nuclear interactions are used to evaluate the material in the Tracker.

10th International Conference on Large Scale Applications and Radiation Hardness of Semiconductor Detectors,

July 6-8, 2011

Firenze, Italy

* Speaker. 


\section{Introduction}

The Silicon Tracker (Tracker hereafter) of the CMS experiment [1] is the largest silicon detector ever built. The inner part is instrumented with pixel detectors arranged in three cylindrical layers in the barrel (BPIX) and two endcaps made of two discs each (FPIX). The outer part is made of silicon strip detectors organized into four independent subdetectors: the Tracker Inner Barrel (TIB) made of four cylindrical layers, the two Tracker Inner Discs (TID) made of three discs each, the Tracker Outer Barrel (TOB) composed of six cylindrical layers and finally the two Tracker EndCaps (TEC) with nine discs each. In total there are 1440 pixel modules providing an accurate two-dimensional measurement, $(r \phi, z)$ coordinates ${ }^{1}$ in the BPIX and $(r \phi, r)$ in the FPIX, and 15148 strip modules providing a one-dimensional measurement, namely the $r \phi$ coordinate in the TIB and TOB and $\phi$ in the TID and TEC. The two inner layers of the TIB and TOB, the two inner rings of the TID, and the first, second, and fifth rings of the TEC are equipped with double-sided modules, with the stereo module sensors tilted by $100 \mathrm{mrad}$, to allow a coarse measurement in the second coordinate. The estimated total mass of the Tracker amounts to about $4150 \mathrm{~kg}$.

The Tracker is successfully taking data since three years, initially collecting atmospheric cosmic ray tracks and then collision events. These data allowed a first comparison between the description of the detector implemented in the simulation and reconstruction software of the CMS experiment and the real detector as it is known from alignment and material budget studies.

\section{Alignment}

The first set of alignment corrections $\left(\mathbf{p}_{m}\right)$ of the full Tracker (geometry in the following) was derived from a track-based alignment performed on a sample of about 3.2 million cosmic ray tracks collected in the Tracker in Fall 2008 [2]. To solve the alignment problem, which consists in minimizing an objective function built using the track-to-hit residuals $\mathbf{r}_{i}$ and the covariance matrix $\mathbf{V}_{i}$ of the measurements

$$
\chi^{2}\left(\mathbf{p}_{m}, \mathbf{q}_{\text {trks }}\right)=\sum_{\text {residuals }} \mathbf{r}_{i}^{T} \mathbf{V}_{i}^{-1} \mathbf{r}_{i}
$$

it was used a combined approach, where a local iterative method ("Hits and Impact Points") [3] was run on the geometry obtained via a global one ("Millepede II") [4]. In the above equation the dependence of the objective function on the tracks parameters $\left(\mathbf{q}_{t r k s}\right)$ is also shown, though these parameters are usually not explicitly determined in the alignment procedure.

As the post-alignment track-to-hit residuals were already dominated by random effects (multiple scattering, intrinsic hit error), the RMS of the distribution of the median of the residuals was chosen as an index of the average off-centering of each module after the alignment procedure. The statistical precision reached was found to be well reproduced by a detailed simulation of the detector (Table 1), for the barrel subdetectors being already close to what was expected in case of no misalignment but with the other conditions of the detector, e.g. the intrinsic hit resolution or

\footnotetext{
${ }^{1} \mathrm{CMS}$ uses a right-handed Cartesian coordinate system with the $x$-axis pointing to the center of the LHC, the $y$-axis pointing upwards perpendicular to the LHC plane and the $z$-axis along the anticlockwise beam direction. When discussing alignment results, a local coordinate system is used for each module, with $u$ being the more precisely measured coordinate, $v$ orthogonal to the $u$-axis and in the module plane, and the $w$-axis normal to the module plane.
} 
the knowledge of the Lorentz angle corrections, assumed to be known with the same uncertainty as in the data. The first geometry obtained from a mixed sample of cosmic rays and proton-proton

\begin{tabular}{|l||c|c||c|c|c|}
\hline \multicolumn{1}{|c||}{} & \multicolumn{2}{c||}{ Cosmic ray tracks } & \multicolumn{3}{c|}{ Collision tracks $(1 / \mathrm{nb})$} \\
\hline & $\begin{array}{c}\text { CRAFT } \\
{[\mu \mathrm{m}]}\end{array}$ & $\begin{array}{c}\text { post-CRAFT MC } \\
{[\mu \mathrm{m}]}\end{array}$ & $\begin{array}{c}\text { post-CRAFT MC } \\
{[\mu \mathrm{m}]}\end{array}$ & $\begin{array}{c}\text { ICHEP2010 } \\
{[\mu \mathrm{m}]}\end{array}$ & $\begin{array}{c}\text { MC not misalign. } \\
{[\mu \mathrm{m}]}\end{array}$ \\
\hline BPIX $\left(u^{\prime}\right)$ & 2.6 & 2.1 & 3.1 & 1.6 & 0.9 \\
BPIX $\left(v^{\prime}\right)$ & 4.0 & 2.5 & 8.9 & 5.5 & 1.8 \\
FPIX $\left(u^{\prime}\right)$ & 13.1 & 12.0 & 10.7 & 5.7 & 2.5 \\
FPIX $\left(v^{\prime}\right)$ & 13.9 & 11.6 & 14.4 & 7.3 & 6.1 \\
TIB $\left(u^{\prime}\right)$ & 2.5 & 1.2 & 10.1 & 5.1 & 3.2 \\
TOB $\left(u^{\prime}\right)$ & 2.6 & 1.4 & 11.1 & 7.5 & 7.5 \\
TID $\left(u^{\prime}\right)$ & 3.3 & 2.4 & 10.4 & 4.0 & 2.4 \\
TEC $\left(u^{\prime}\right)$ & 7.4 & 4.6 & 22.1 & 10.1 & 2.9 \\
\hline
\end{tabular}

Table 1: RMS of the distributions of the median of the residuals (DMR) for the different Tracker subdetectors. The two columns in the left part refer to an alignment performed with the same strategy on a sample of real ("CRAFT") and simulated ("post-CRAFT MC") cosmic ray tracks. The three columns in the right part refer to a sample of minimum bias tracks and represent: DMR expected from simulation using an alignment based only on cosmic ray tracks ("post-CRAFT MC"), determined in data after an alignment done mixing cosmic rays and collision tracks ("ICHEP2010") and expected from simulation in case of no misalignment (“MC not misalign.”).

collision tracks was produced after $1 / \mathrm{nb}$ of integrated luminosity and it improved considerably the statistical precision also in the forward subdetectors. Being already close to the statistical precision limit, most of the studies were then focused in analyzing and monitoring the geometry of the Tracker and investigating possible sources of systematic biases. The main features of the Tracker geometry which have been observed are:

- the two halves of the TIB (forward/backward) are separated along the $z$ direction of about $5 \mathrm{~mm}$. This gap was already found in the analysis of the cosmics collected in Fall 2008 (Figure 1) and was confirmed by the optical survey measurements;

- the two halves of the BPIX (left/right) are observed to move relative to each other by up to $90 \mu \mathrm{m}$ in the $z$ direction, in discrete movements. These movements were determined by the unbiased track-to-primary vertex residuals ${ }^{2}$ and recovered by performing the alignment procedure in several separate periods (Figure 2). No clear correlation with the operations of the detector, like the switching on and off of the cooling system or the cycling of the superconducting CMS solenoid, has been found so far;

- the overall Tracker is tilted with respect to the direction of the magnetic field of the superconducting CMS solenoid (Figure 3). The tilt angles are determined as those rotations around

\footnotetext{
${ }^{2}$ This method consists in selectively removing a track from the event, determine a primary vertex with the others, compute the transverse and longitudinal projections of impact parameter of the probe track with respect to the primary vertex and finally studying the mean value of the distribution of the residuals as a function of $\phi$ and $\eta$ of the track.
} 
the $x$ - and $y$-axis optimizing the track quality estimated from the average $\chi^{2}$ probability of the track fit. The tilt angle around the $x$-axis is found to be $\theta_{x}=300 \mu \mathrm{rad}$ while that around the $y$-axis is compatible with a null angle.

- the silicon surface in the modules is not flat (Figure 4) as indicated by the deterioration of the probability of the $\chi^{2}$ of the track fit for tracks with large impact angle with respect to the normal to the surface of the module. Sensors are bowed with sagittas up to $30 \mu \mathrm{m}$ and modules made of two sensors have kinks of $1.6 \mathrm{mrad}$ on average.

All these effects are mechanically allowed and have been corrected for by the alignment procedure. On the other hand these effects are not yet included in the detailed geometry of the Tracker used in the simulation, as their impact, in terms of loss of hits or poor description of the regions with overlapping modules, was found so far to be negligible.

\section{Material Budget}

The description of the Tracker in the simulation package of the CMS experiment consists of more than 350000 GEANT4 volumes. In Table 2 [5], a breakdown of these volumes into categories of homogeneous materials, likely to be known after the assembly with a similar accuracy, indicates that the silicon sensors, which are the most accurately known components of the Tracker both in term of their mass and of chemical composition, contribute to the total mass of the detector only by about 5\%. The largest contribution to the Tracker mass is by far represented by the passive structures for which the uncertainty on their overall amount in the real detector is the largest. Furthermore, in the software description of the Tracker, the passive volumes are usually filled by average mixtures of their components. This introduces the necessity of some approximation in the description of the detector. On the other hand the overall material crossed by a particle traversing the Tracker volume can exceed $1 X_{0}$ and $0.5 \lambda_{I}$, with $X_{0}$ and $\lambda_{I}$ being the radiation and the nuclear interaction lengths respectively, so a small fractional uncertainty on the description of the material can have a sensible impact on the expected physics performance. Detailed studies of the material budget of the Tracker have been conducted since the first collisions [6], by reconstructing the interactions of the radiation with the material of the Tracker itself:

- photon conversions: about $70 \%$ of all the photons convert into an $e^{-} e^{+}$pair within the Tracker volume. The vertices of the conversions, reconstructed with a typical spatial resolution $\sigma_{r}=0.2-0.5 \mathrm{~cm}$, can be used to probe the $X_{0}$ distribution of the material.

- nuclear interactions: at $p_{T}=5 \mathrm{GeV} / c$, about $5 \%$ of the charged pions interact in the Tracker. The vertices of the interactions, reconstructed with a typical spatial resolution $\sigma_{r}=0.01 \mathrm{~cm}$, can be used to probe the $\lambda_{I}$ distribution of the material.

Both processes are characterized by a secondary (interaction) vertex displaced from the primary vertex of the event and feature outgoing soft not-pointing tracks. Therefore, for the study of the material budget two additional tracking steps, aimed to increase the number of the reconstructed conversions outside the pixel volume, were developed and added to the standard track reconstruction sequence used in CMS. Results described below were obtained on a sample corresponding 

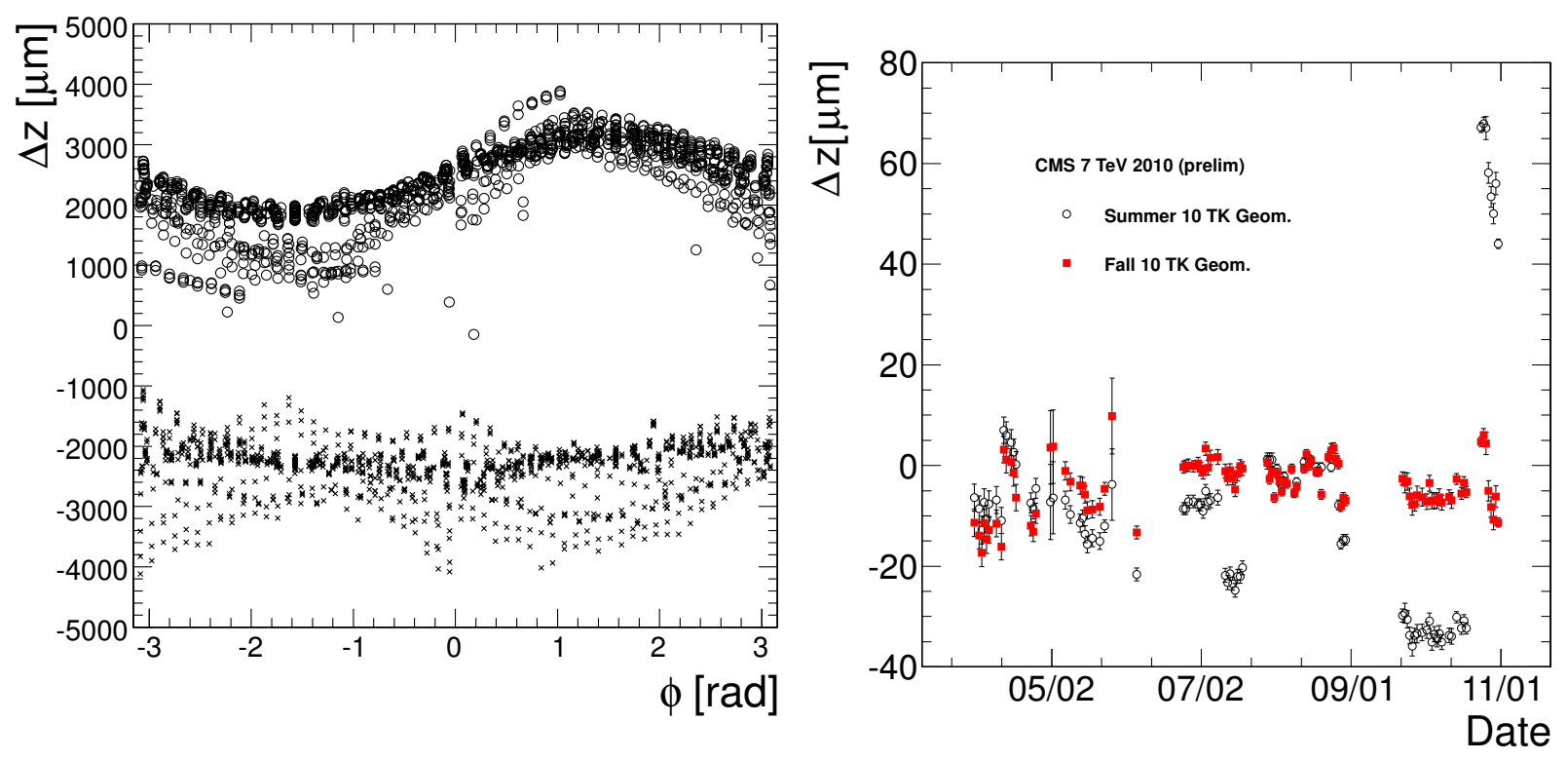

Figure 1: Comparison of the position of the TIB modules determined by the alignment with cosmic ray tracks with respect to the design one, $\Delta z=z_{\text {design }}-$ $z_{\text {cosmics }}$, as a function of $\phi$. Crosses (circles) represent modules of the forward (backward) TIB halfbarrel [2].

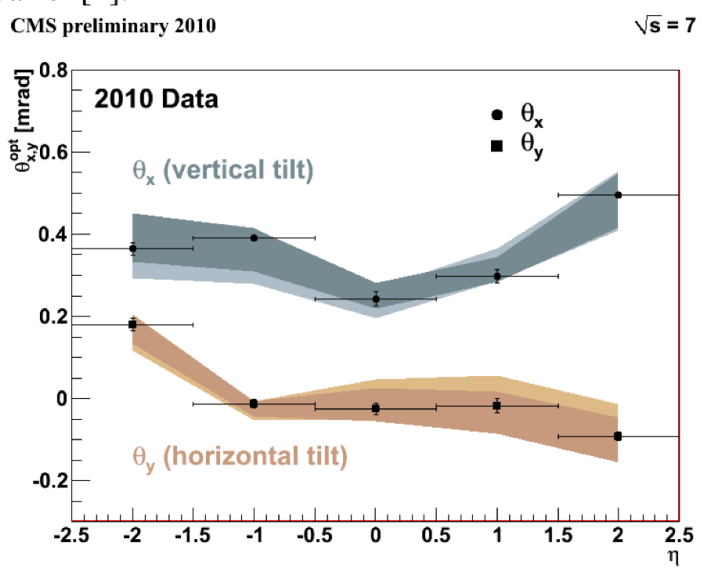

Figure 3: Tilt angles $\theta_{x}, \theta_{y}$ of the Tracker around the $x$ - and $y$-axis optimizing the average $\chi^{2}$ probability of tracks in minimum bias events. Angles are shown in bins of the track pseudo-rapidity $\eta$. The shadowed bands represent the systematic uncertainty estimated modifying the acceptance criteria of the tracks (from $p_{T}>0.5 \mathrm{GeV} / c$ to $\left.p_{T}>2 \mathrm{GeV} / c\right)$ and the quantity used to estimate the track quality (dark shade is the RMS, light shade is the minimum maximum range of excursion).

Figure 2: Separation in $z$ of the BPIX half-barrels as estimated from the unbiased track-to-primary vertex residual method as a function of time for the 2010 LHC proton-proton run. Empty (filled) dots are the pre-(post-)alignment values.

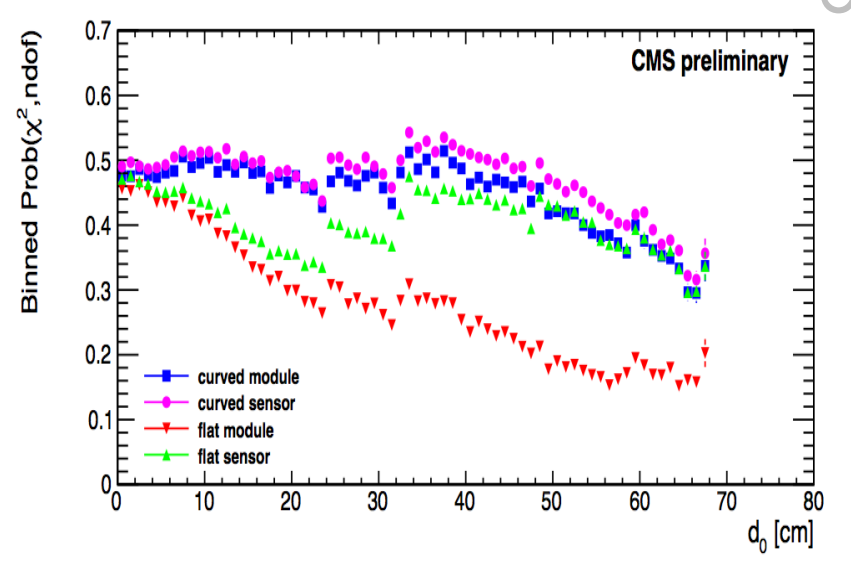

Figure 4: Average $\chi^{2}$ probability of the track fit as a function of the closest distance $d_{0}$ of the track to the nominal beamline for cosmic ray muons, for different assumptions for the surface of the silicon modules. In the legend, "module" indicates that the assumption on the shape of the measurement surface ("flat" or "curved") was made on the module as a whole while "sensor" means that in two-sensors modules each of them was considered separately. 


\begin{tabular}{|l|r|r|}
\hline Component Category & Mass $[\mathrm{kg}]$ & Fraction [\%] \\
\hline Carbon Fiber & 1144.5 & 27.6 \\
Copper and copper alloys & 644.5 & 15.6 \\
Aluminium & 595.0 & 14.4 \\
Organic materials & 472.1 & 11.4 \\
Coolant $\left(\mathrm{C}_{6} \mathrm{~F}_{14}\right)$ & 258.9 & 6.3 \\
Silicon sensitive & 225.8 & 5.5 \\
Fiber-glass laminated & 213.4 & 5.2 \\
Other mechanical structures & 191.7 & 4.6 \\
Inorganic oxides & 153.2 & 3.7 \\
Other metals & 141.9 & 3.4 \\
Glues and resins & 75.3 & 1.8 \\
Electronic components & 25.7 & 0.6 \\
\hline
\end{tabular}

Table 2: Mass per material category in the GEANT4 description of the Tracker volume. A detailed description of the components included in each category is given in [5].

to $1 / \mathrm{nb}$ of integrated luminosity where about 260000 photon conversions and 470000 nuclear interactions were reconstructed.

Ideally material budget studies should provide a map of the detector material to be compared with the description implemented in the GEANT4 simulation. Quantitative comparisons between data and simulation are first performed on radial distributions, e.g. obtained integrating over the azimuthal angle. Two main corrections are then required to transform the distributions of reconstructed interaction vertices into distributions of material. First of all, it is known from tracking and alignment studies that in the transverse plane the center of the BPIX is largely offset with respect to the beamline, approximately of $x_{b l}-x_{B P I X}=0.23 \mathrm{~cm}$ and $y_{b l}-y_{B P I X}=0.38 \mathrm{~cm}$. The offset between the beamline and the beam pipe is instead much smaller, about $100 \mu \mathrm{m}$. In the simulation the same offset between the beamline and the center of the BPIX as measured in the data is introduced to reproduce the non uniform illumination in $\phi$ of the BPIX; the offset between the centers of BPIX and of the beam pipe is neglected instead. Figure 5 shows the radial distribution of the nuclear interaction vertices for two different choices of the point with respect to which the radial distance in the data is defined: the peaks corresponding to the three BPIX layers are smeared out when the center of the beam pipe is used as reference and the opposite happens for the beam pipe peak when the center of the BPIX is used as reference point. The effect becomes less important at larger radii and in the following the radial distances are defined with respect to the BPIX center. Maps of the material, to be compared with the GEANT4 description, are extracted from the number of reconstructed nuclear interaction vertices in a given volume $V\left(N_{n . i .}^{\text {raw }}\right)$ translated into an average interaction probability in that volume after subtracting the number of fake interactions $\left(N_{n . i .}^{f a k e}\right)$, correcting for the efficiency of reconstructing a nuclear interaction $\left(\varepsilon_{n . i .}\right)$ and normalizing to the number of hadrons expected in $V$ for the sample of considered events $\left(f_{\text {geom,had }}\right.$ and $N_{\text {evts }}$ 

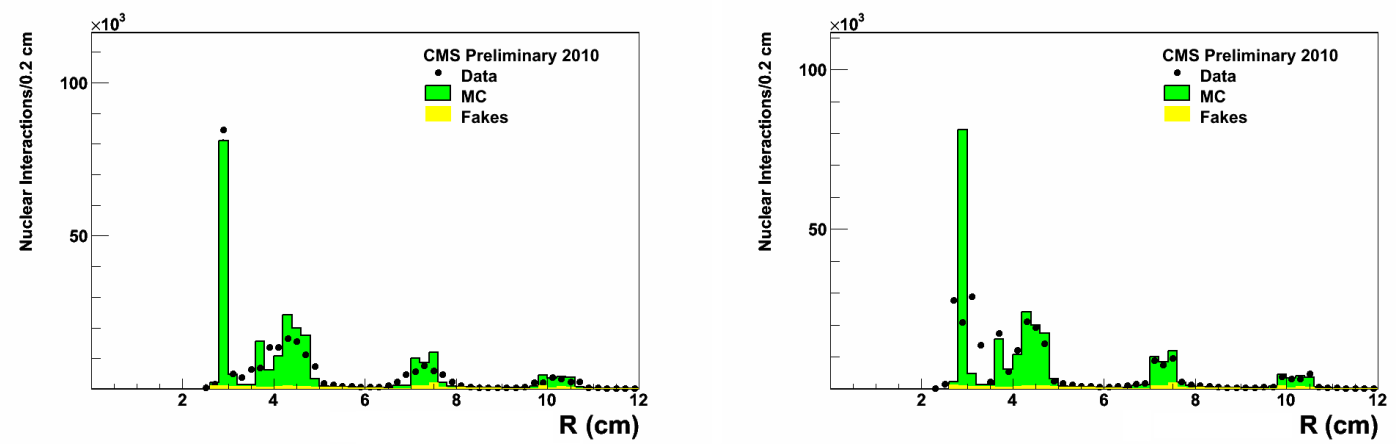

Figure 5: Distribution of the radial distance, in the transverse plane, for nuclear interaction vertices in data and simulation in the BPIX region: distance in the data is computed either with respect to the beam pipe center (left) or with respect to the BPIX center (right) [6].

respectively):

$$
\left\langle\frac{1}{\lambda_{I}}\right\rangle \propto \frac{1}{N_{\text {evts }}} \frac{N_{n . i .}^{\text {raw }}-N_{n . i .}^{\text {fakes }}}{\varepsilon_{\text {n.i. }} f_{\text {geom, had }}} .
$$

A similar relation holds for the average $\left\langle 1 / X_{0}\right\rangle$ from the number of reconstructed photon conversions. The radial distributions of the average $\left\langle 1 / X_{0}\right\rangle$ and $\left\langle 1 / \lambda_{I}\right\rangle$ are shown in Figure 6. There is a good agreement both between the average quantities determined in the data and in the simulation and with the simulation truth from the GEANT4 model of the Tracker. More quantitative studies indicate an agreement at the $10 \%$ level with the exception of the region between the TIB and the TOB where there is a $20 \%$ excess in the data. Figure 7 shows the $x-y$ map of the material distribution, zoomed into the BPIX region, $|z|<26 \mathrm{~cm}$, in terms of $\left\langle 1 / \lambda_{I}\right\rangle$ in the GEANT4 model and as determined from the reconstruction of nuclear interactions in the data. The GEANT4 volumes describe quite accurately the real detector apart from few structures (details in the supporting rails of the BPIX and a pair of carbon fiber stiffeners) which are missing. Furthermore in the data the off-centering of the the beam pipe with respect to the BPIX is clearly seen.

\section{Conclusions}

A key ingredient of the excellent performance of the Tracker of the CMS experiment has been the continuous effort in understanding the accuracy of the description, in the simulation and reconstruction software, of both its active and passive components. This effort started during the commissioning phase and has produced a first set of benchmark results after the first $1 / \mathrm{nb}$ of proton-proton collisions were recorded. Currently more than $1 / \mathrm{fb}$ of integrated luminosity has been collected by the CMS experiment. The analysis of this large sample will allow to further improve the usage of the real Tracker as a powerful scientific instrument.

\section{References}

[1] CMS collaboration, The CMS experiment at the CERN LHC, 2008 JINST 3 S08004. 

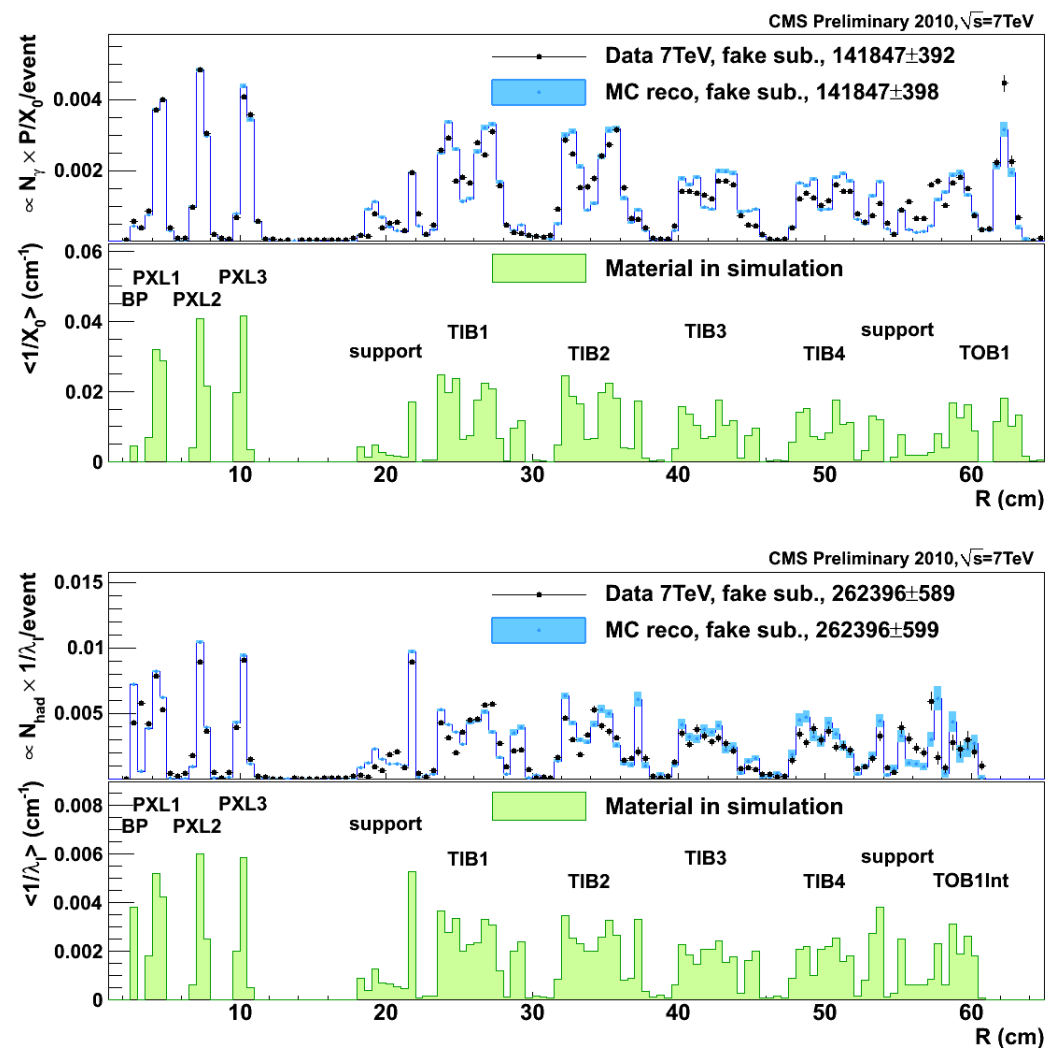

Figure 6: Distribution of the material versus the radial distance, estimated from photon conversions (top) and from nuclear interactions (bottom). In the bottom part of each panel, the average values per bin in the GEANT4 description are shown [6].
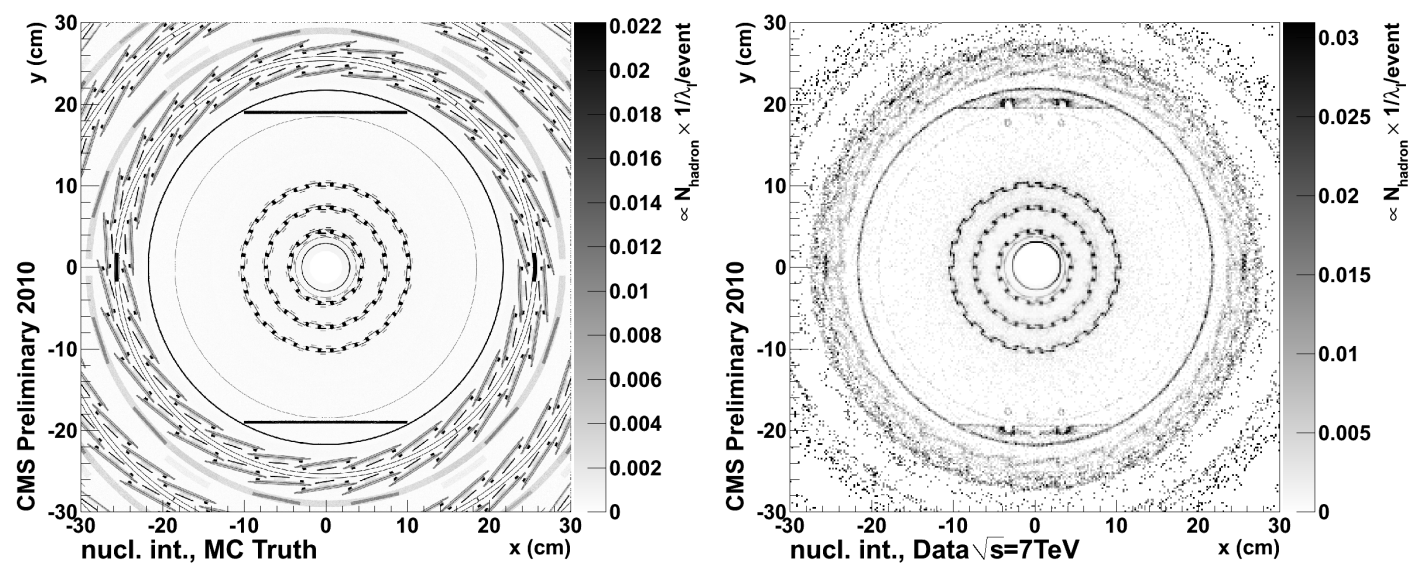

Figure 7: Maps of the material distribution for the nuclear interactions in the $x-y$ plane, zoomed into the BPIX region $(|z|<26 \mathrm{~cm})$ : simulation truth with $0.5 \times 0.5 \mathrm{~mm}^{2}$ bin size (left) and reconstructed distribution in data with $0.2 \times 0.2 \mathrm{~cm}^{2}$ bin size (right) [6]. 
[2] CMS Collaboration, Alignment of the CMS silicon tracker during commissioning with cosmic rays, 2010 JINST 5 T03009 [arXiv:0910.2505].

[3] V. Karimäki, T. Lampén and F.P. Schilling, The HIP algorithm for track based alignment and its application to the CMS pixel detector, [CMS-NOTE-2006-018].

[4] V. Blobel, Software alignment for tracking detectors, 2006 NIMA 566 5;

G. Flucke, P. Schleper, G. Steinbrück and M. Stoye, CMS silicon tracker alignment strategy with the Millepede II algorithm, 2008 JINST 3 P09002.

[5] E. Migliore, G. Sguazzoni, Altered scenarios of the CMS Tracker material for systematic uncertainties studies, [CMS-NOTE-2010-010].

[6] CMS Collaboration, Studies of Tracker Material, [CMS-PAS-TRK-10-003]. 